\title{
Design Information Management for Product Sound Quality: Requirement Definition
}

\author{
Kazuko Yamagishi ${ }^{1}$, Koichi Ohtomi ${ }^{2}$, Kenichi Seki ${ }^{2}$, and Hidekazu Nishimura ${ }^{1}$ \\ ${ }^{1}$ Graduate School of System Design and Management, Keio University, Japan \\ ${ }^{2}$ SDM Lab, Graduate School of System Design and Management, Keio University, Japan \\ kaz-yamagishi@a6.keio.jp
}

\begin{abstract}
In a current design information management of consumer products, it is difficult to take into consideration of the sensory preference of users in requirements definition phase. Especially, sensory preference on sound of products might be different individually and depend on environment where the products are used. The engineering metrics are necessary to be connected to the sensory preference of users to design the right products to user preferences. In this paper we propose a process to manage design information considering product sound quality, and investigate a consistent description method using SysML (Systems Modeling Language) from requirement definition to engineering metrics setting. Picking up a camera as an example of consumer products we extract customer demands using the evaluation grid method according to the cases where the camera is used. By applying the DSM (Design Structure Matrix) clustering analysis to the results of the interview, we cluster the fundamental requirements relating functional and performance requirements which include sound quality. Also a stepwise refinement from the requirement definition to the architectural design is performed using SysML diagrams, and the appropriate engineering metrics are derived.
\end{abstract}

Keywords: sound quality, SysML, evaluation grid method, use case, DSM clustering analysis, Product Lifecycle Management.

\section{$1 \quad$ Introduction}

In recent years, the value of product sound quality is increasing, and many researches for sound design have been conducted. Ohtomi et al. (2008) considered that sound quality could add values to product. Yanagisawa et al. (2009) proposed a systematic approach which guides from the definition of requirements to functional and structural design in terms of product sound. Sound demands for product may vary depending on use cases, and it is difficult to set sound quality target which matches the customer needs. Thus, it is not easy to integrate the sensory preference such as sound as system requirements using the existing design-information management. Since the sound design process needs to carry over the sound demands to multiple 
design domains, the common understanding of the sound-related design information among the engineers are essential.

In a recent study of product design processes, SysML (Systems Modeling Language) have been utilized as a common notation for the system model (Balmelli et al. 2007; Zhu et al. 2009; Seki et al. 2009). However, few studies using SysML manage the demands about sensory preference depending on use cases as far as authors know.

By using the evaluation grid method (Kelly, 1995), we have already drew out customer demands for digital cameras depending on their use cases (Yamagishi et al. 2013). In addition, we have proposed to extract requirement clusters by applying DSM (Design Structure Matrix) clustering analysis (Eppinger et al. 2012) to the result of the evaluation grid method test. Through these processes, the demands for the product and its sound demands have become clear, and finally we have set the subjective sound quality target according to each use case.

In this paper, we investigate the description method of a system model using SysML, which can clarify sound demands as system requirements and to carry over the design information to multiple design domains. First, in the process of requirement analysis, the functionality which a system provides, and its relationship with actors around the product are clarified by using use case diagram. To clarify the function of the system, we describe use cases using sequence diagram and extract the interaction between a system and actors. Then the requirement diagram, which associates the functional requirements and the sound demands, is created and further the functional requirements are associated with the requirement clusters obtained from the evaluation grid method test and DSM clustering analysis. The requirement diagram ensures traceability between demands for a camera as a product and the functional and performance requirements.

In the process of functional design, sequence diagram is used in order to clarify the function of emitting shutter sound. The components of the camera are defined in block diagram. Behavior of the system components relevant to the sound are clarified using the activity diagram. The physical engineering metrics are allocated to these components, and values of the engineering metrics of each components are defined using proper simulation models. Through the proposed process, subsequent structural design can be proceeded effectively using the engineering metrics to realize the product that satisfied sensory customer demands.

\section{SysML Based Representation of Sound Design Information}

\subsection{Requirements Transfer Process}

In order to integrate the sensory preference such as sound quality as system requirements as well as functional requirements, and carry them over multiple design domains, we propose requirements transfer process as shown in figure 1.

In conventional camera design, requirement analysis is conducted mainly focusing on functional requirements such as taking an image. In the proposed process of 
requirements analysis, first, a use case diagram is used to clarify the function of a product system and the relationship with other participants around the product. Then, next, multiple sequence diagrams describe interactions among actors for every use case, and they detail interaction including sound related events. Through a stepwise refinement in functional and structural design of this process, sound quality requirements are transferred to the appropriate engineering metrics.

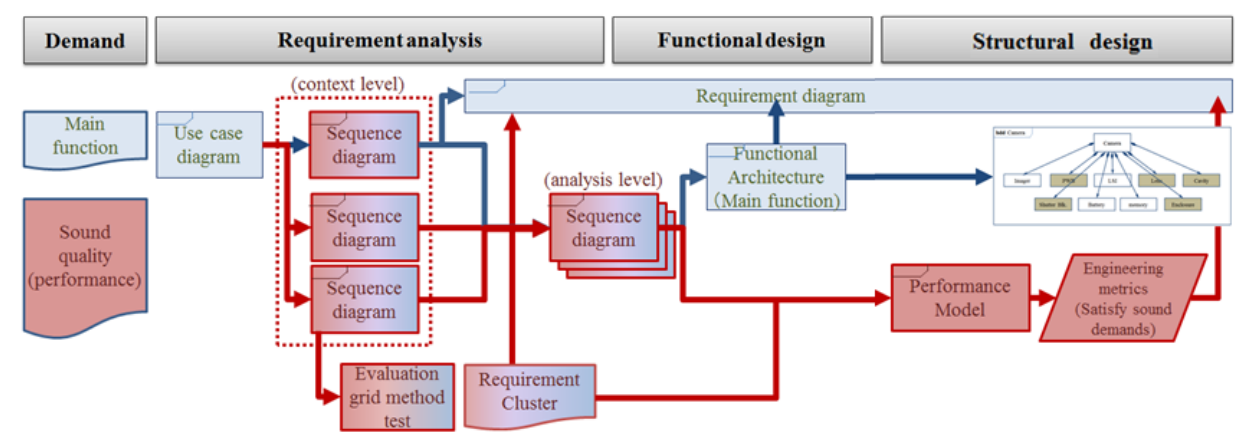

Fig. 1. Requirements transfer process

\subsection{Clustering Analysis for Requirements Using the Evaluation Grid Method [7]}

In order to capture demands for product which varies depending on situations, we carry out an evaluation test using the evaluation grid method that assumed use cases. Evaluation grid method is the personal interview method based on alternative constructivism and psychological theory by G.A. Kelly (1995). This methodology enables us to clarify recognition structure of value or attractiveness for the subject.

Figure 2 shows steps to extract requirement clusters from the test using the evaluation grid method. Following steps were taken: In STEP 1, we asked the participants about their preferred products and reasons. We determined the term that the participants used when comparing the products as 'Evaluation points'. 'Evaluation points' are the criteria used by the participants to assess the products' relative superiority, and thus can be interpreted as demands for product. Next, the participants were asked why the 'Evaluation points' inspired their choice. This rudder-up step was performed to lead higher demands. We determined the term used to describe the reason as 'Reasons (WHYs)'. Similarly, a rudder-down step was performed to lead concrete measures for each evaluation point. We determined the term used to describe the measures as 'Concrete measures (HOWs)'. In STEP 2, three requirementsevaluation points, reasons and concrete measures were assigned as items in the rows and columns of the DSM. The circular symbol $(0)$ in the matrix represents the correlation between requirements in the rows and in the columns in DSM. The sound symbol (S) represents the requirement correlation in terms of sound. In STEP 3, to 
find the group of highly correlative requirements, the clustering analysis was used. From requirement clustering analysis, the requirements were interpreted in each cluster, and thus we can capture the structure of demands for product. When sound demands ( $\mathrm{S}$ symbol) are included in a cluster, it indicates that realizing sound demands can satisfy demands for product of the cluster. The requirement diagram for a camera is created referring to this DSM clustering result.

STEP 1. Evaluation grid method network

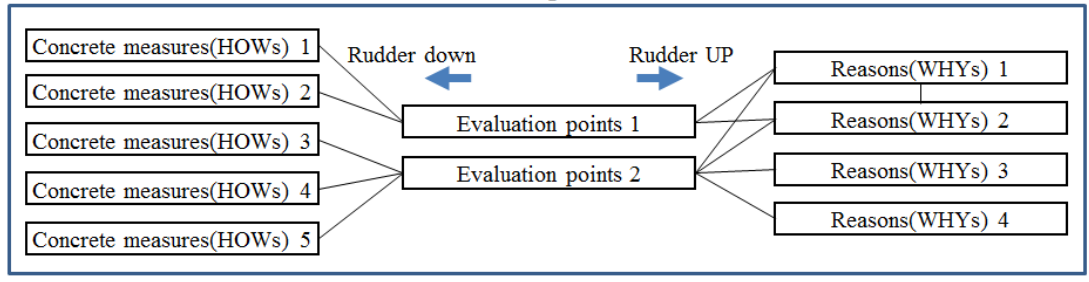

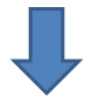

STEP2. Converting to DSM

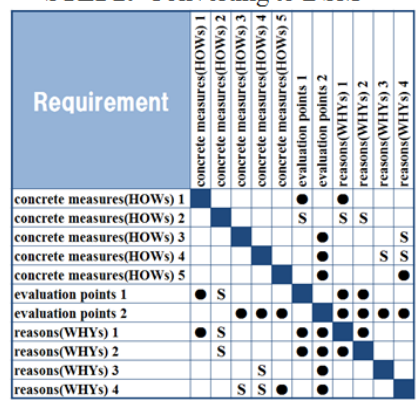

STEP3. Requirement Cluster

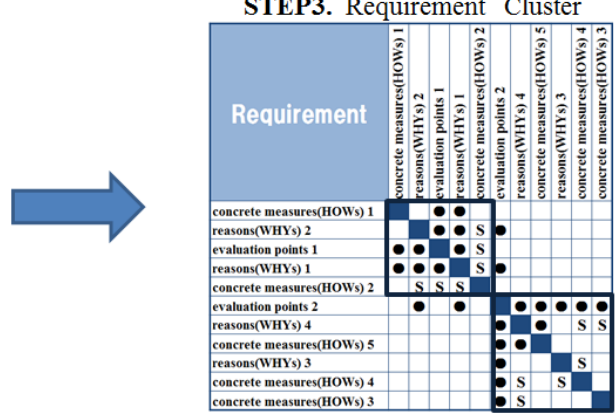

Fig. 2. DMM for requirement clusters

\subsection{Functional and Structural Design for Performance Requirements}

From the requirement clustering analysis, functional requirements to the system and sound demands are classified, and the structure of requirements becomes clear. To realize sound demands physically, a function of sound is examined. A sequence diagram is used to clarify the functions of emitting shutter sound. From the sequence diagram, interaction between each component relevant to sound such as vibration force and sound wave become clear. Next, the components of a camera which have connections with the function of sound, such as the shutter block and the cavity, are identified. To visualize and organize the physical energy flow about sound and vibration among these components, activity diagram is used. Thus, engineering metrics relevant to each component is derived. Since the sound demands for every use case is clear as a sound quality target, a product sound quality can be achieved by 
considering the shutter mechanism which realizes the desired engineering metrics by simulation.

\section{$3 \quad$ Application to Camera Shutter Sound}

\subsection{Use Cases for a Camera}

This section illustrates the application for the shutter sound design. As for the camera shutter sound is required for customers to know whether the product is functioning normally. However, the sound may be unnecessary in a quiet place. Customer demands for shutter sound of a camera may vary depending on use case. The use case diagram of 'taking photo' is shown in Fig. 3. From the viewpoint of sound design which considers the impact of shutter sound to other persons in a quiet place, it is necessary to think 'the surrounding people' who may hear shutter sound other than the user and subject people as an actor. About three camera use cases; record a photographic subject, take a photograph of landscape, and take a photograph at the quiet place, sequence diagrams describe interactions among actors and are shown in Fig. 4.

By describing use cases with sequence diagrams, it became clear how sound is concerned with actors associated with main function of a camera 'taking photograph' depending on each use case. Moreover, the visual contents for the evaluation grid method test could be decided according to above-mentioned investigations using use case and sequence diagrams, and the questionaries' contents about sound were also able to be prepared without shortage.

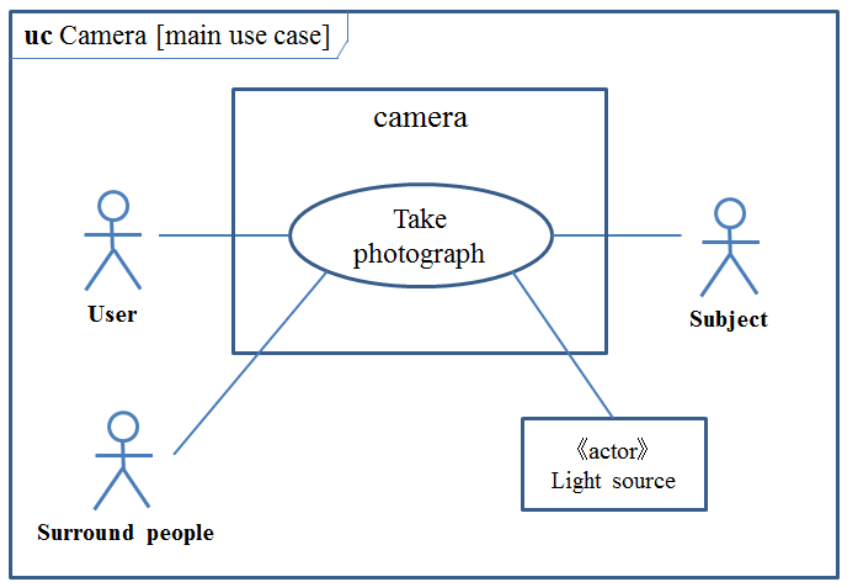

Fig. 3. Main use case of a camera 

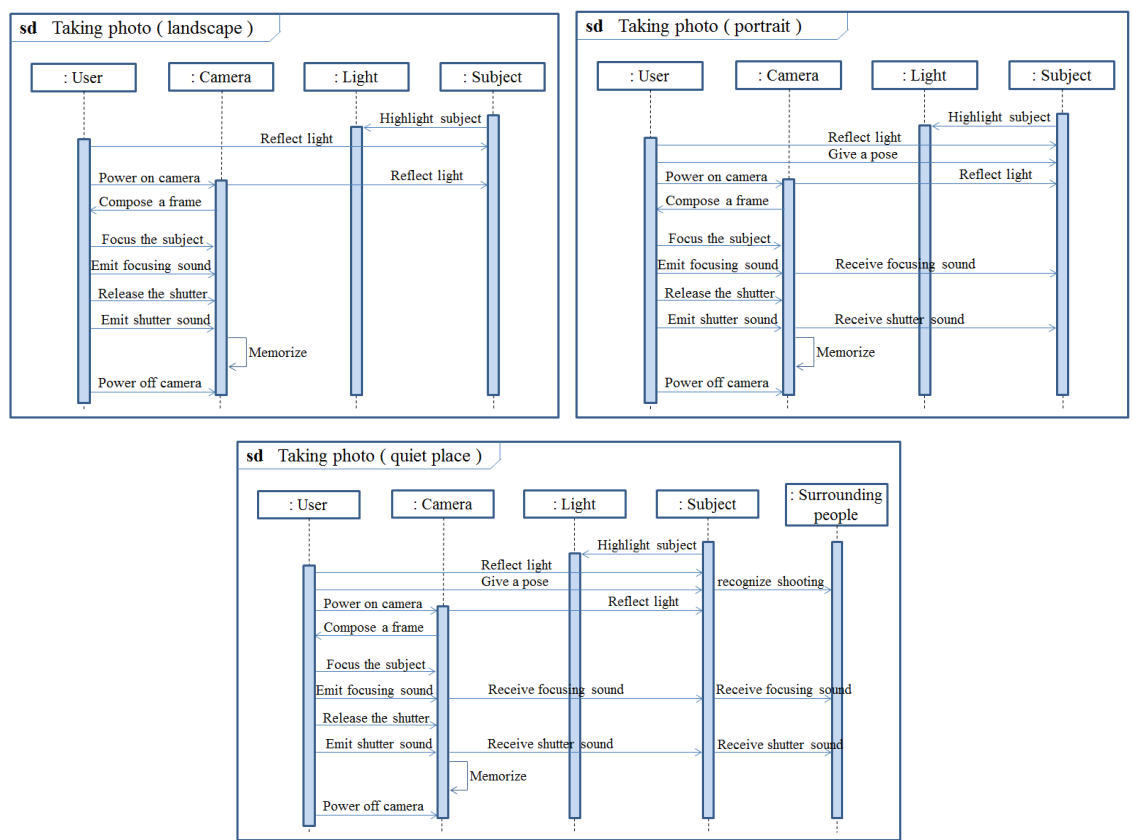

Fig. 4. Sequence diagram assuming use cases

\subsection{The Requirement Analysis Using the Evaluation Grid Method for Camera}

We conducted the evaluation test of camera using the evaluation grid method with about ten interviewees. The participants used five evaluation cameras with screen images, which assuming use cases decided by the method described in section 3.1 to determine customer demands depending on use cases. Specifically, to make them feel as if they are present in the scene, they operated all cameras freely in front of four types of video pictures by a $4 \mathrm{~K}$ projector. The four scenes included an athletic meet, magnificent scenery, the bustle of the city and animals. We interviewed the participants about their preferred product and the reasons.

As shown Fig. 5, DSM data were constructed as described in section 2.2 using evaluation grid method. Through this process, close requirements were concentrated, making it easier to understand demands for product. Customer demands for the camera are clarified into the following six requirement clusters.

1) Pleased with clicking the shutter

2) Use the camera casually, fashionably

3) Enhance their cool look by possessing and operating the camera

4) Feeling of comfort in taking photos as will

5) Quiet sound that matches the scenes and circumstances

6) Regard the camera's functionalities to take photos as import 


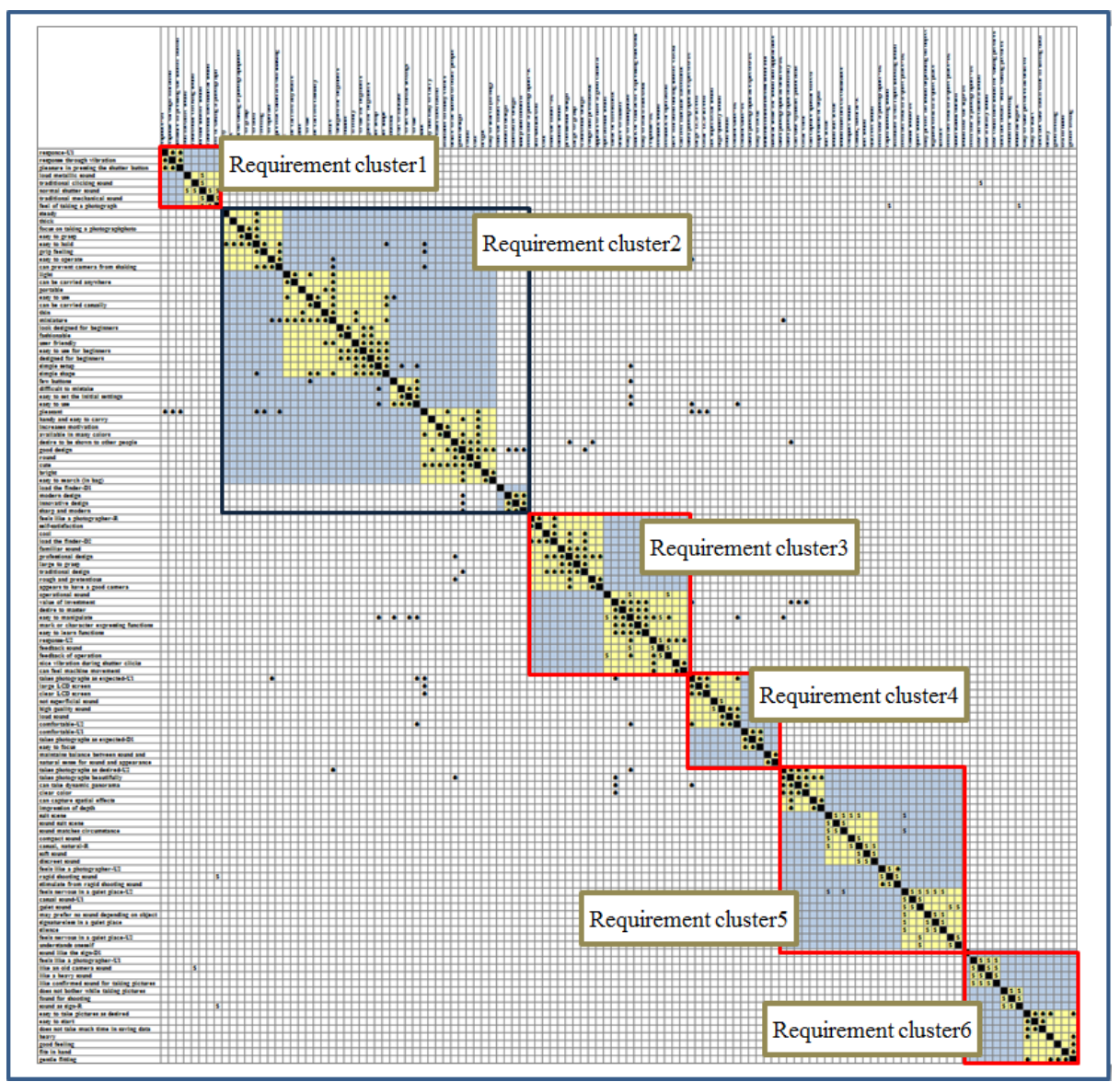

Fig. 5. DSM for Requirement cluster

First, we analysed system requirements focusing main function of a camera by drawing requirement diagram. And, the sound demands derived from the requirement clusters are added to the requirement diagram of a camera. The items which were highlighted gray were added after using the requirement clustering analysis. This resulted requirement diagram is shown in Fig. 6. For example, the demands about quiet sound which does not cause circumference problem is a requirement which did not come out unless we performed the evaluation grid method test. Thus, the demands which are not visible from usual requirement definition become clear from requirement clusters using evaluation grid method test. Furthermore, it revealed the relationship between the sound demands and demands for a camera. Therefore, We can trace demands for the camera are came out of which cluster and can know the sound to satisfy the demands from the cluster., and we can share the demand structure among the people in associated development organizations. 


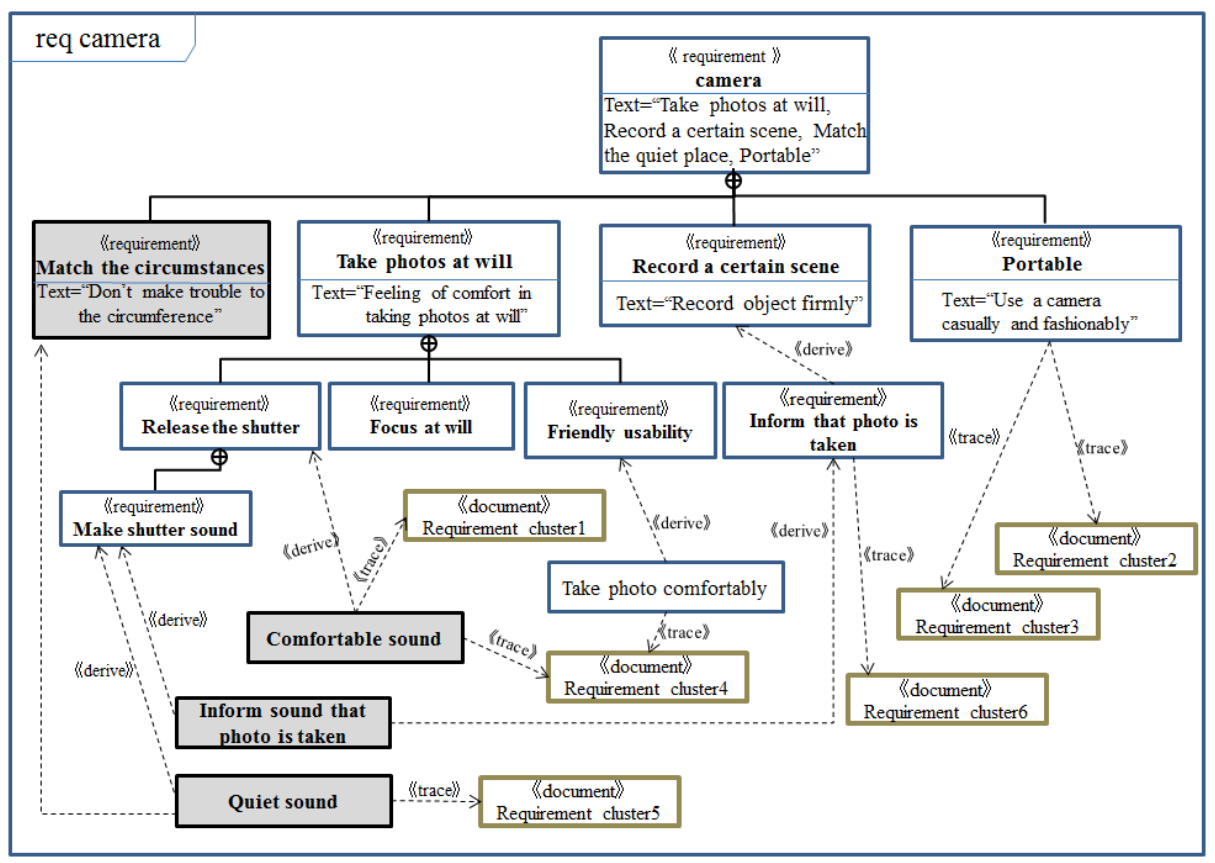

Fig. 6. Requirement diagram for a camera

\subsection{Functional and Structural Design for a Camera}

From the requirement diagram described in section 3.2, demands for a camera and the sound demands became clear. In order to clarify the function of sound, sequence diagram is used. The sequence diagram of emitting shutter sound is shown in Fig. 7. As shown in Fig. 7, interaction between each component relevant to sound such as vibration force and sound wave can be recognized during the shutter release behavior from the observation of sequence diagram.

Next, the block diagram of the camera components which realize the functions of a camera is shown in Fig. 8. The items which are highlighted gray are the components which have a connection with the function of sound. Activity diagram shown in Fig. 9 visualize the physical energy flow about sound and vibration among these components. Paying attention to a 'shutter block', 'Cavity', etc. which is the module structure relevant to sound, engineering metrics relevant to each module is derived.

Since the sound demands for every use case is clear as sound requirements, the demands can be realized by considering the performance requirements which are quantified as engineering metrics. The value of the engineering metrics is determined based on the sound quality targets assigned as system requirements. The relationship between engineering metrics and the structural and acoustic vibration mechanism relevant to shutter sound is shown in Fig. 10. The product sound quality can be achieved by designing the shutter structures with the engineering metrics. 


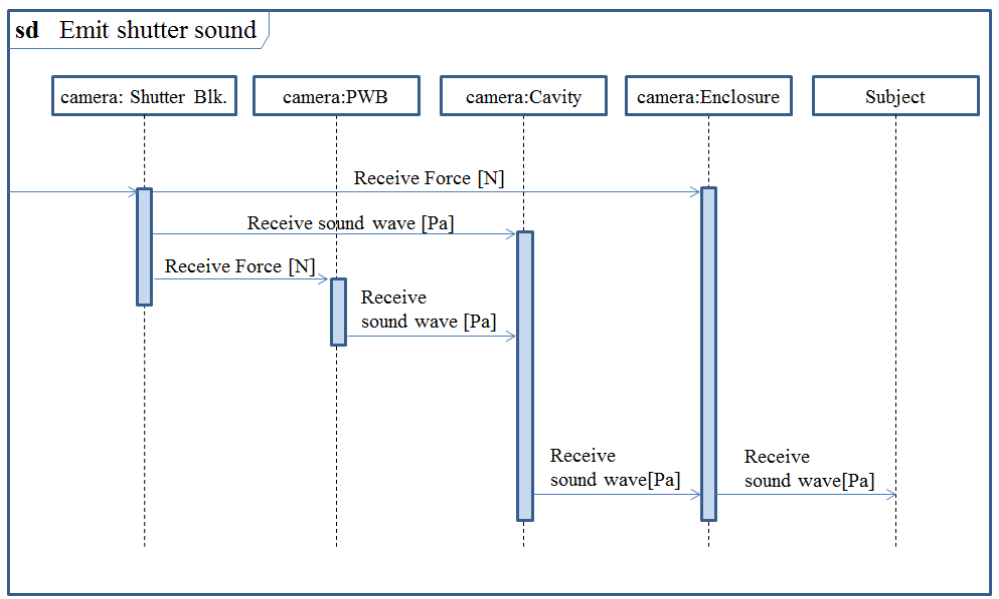

Fig. 7. Sequence diagram of 'Emit shutter sound'

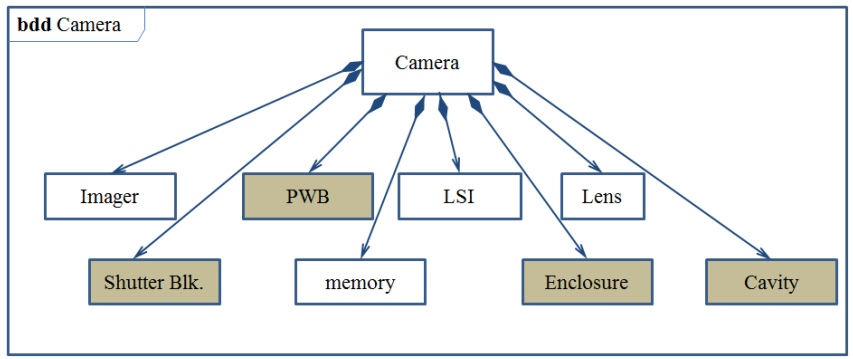

Fig. 8. Definition block diagram of a camera

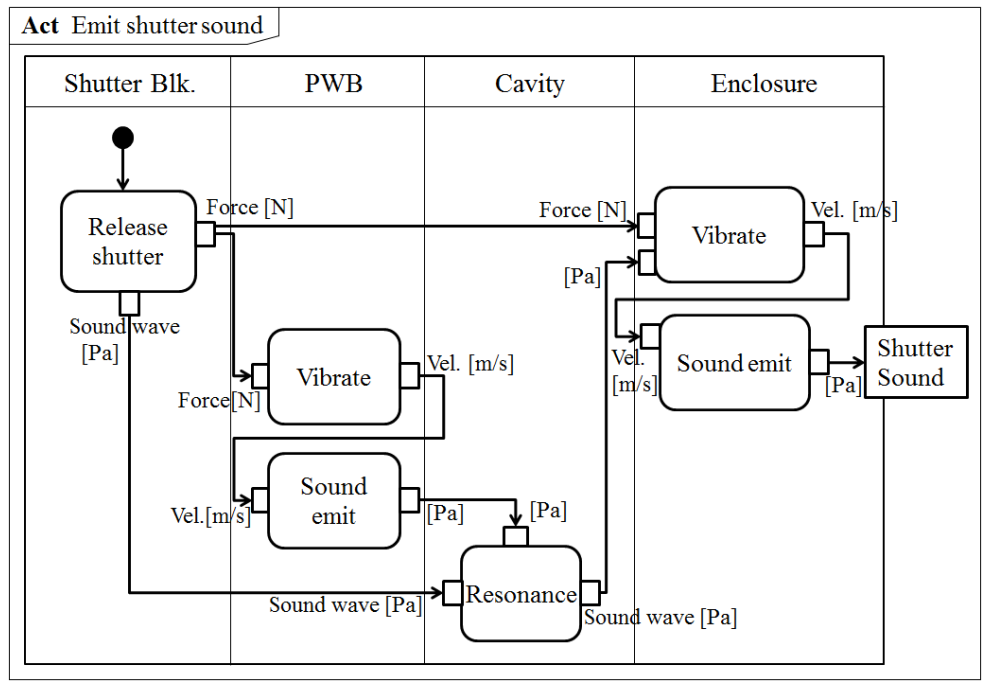

Fig. 9. Activity diagram of 'Emit shutter sound' 


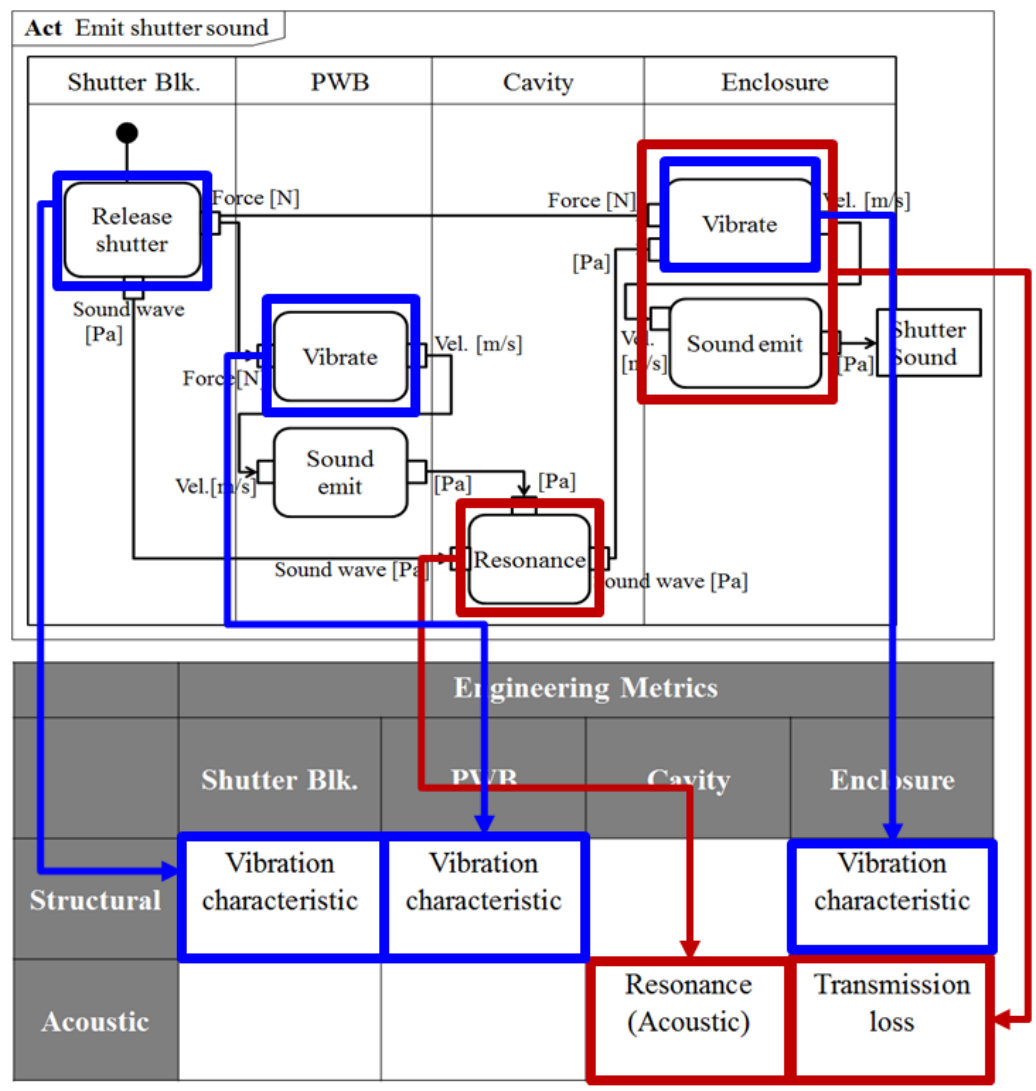

Fig. 10. Correlation of engineering metrics and components

\section{Conclusion}

In this paper, we propose a process to manage design information considering product sound quality. And we investigate the description method of a system model using SysML in order to clarify sound demands as system requirements and to carry over the design information about sound demands to different product development organizations. Sensory preference on sound of products might be different individually and depend on environment where the products are used. Therefore, based on the use case investigations, the customer demands were explored thoroughly by the evaluation grid method. DSM clustering was performed to the result of the evaluation grid method test, and the requirement cluster was extracted. Through the requirement clustering analysis, it is possible to set sound target depending on use cases. Then the requirement diagram, which associates the functional requirements and the sound demands, is created and further the functional requirements are associated with the requirement clusters obtained from the evaluation grid method test and DSM clustering analysis. Thus, the demands which are not visible from usual 
requirement definition become clear. And the requirement diagram ensures traceability between demands for a camera as a product and the functional and performance requirements.

In order to realize sound demands physically, a function of sound was examined. A sequence diagram is used in order to clarify the function of emitting shutter sound, and identify the interactions among component relevant to sound. To visualize and organize the physical energy flow about sound and vibration, activity diagram was used. Thus, engineering metrics relevant to each component were derived and stored as performance requirements. Through a stepwise refinement in functional and structural design, sound quality requirements are transferred to the appropriate engineering metrics. This process can help design teams to realize successful product that satisfied sensory customer demands.

The developed SysML system model considering product sound quality can be used in the test phase of the product at manufacturing sites. This sound design process is helpful for design teams in terms of product lifecycle management.

\section{References}

1. Balmelli, L.: An Overview of the Systems Modeling Language for Products and Systems Development. The Journal of Object Technology 6(6), 149-177 (2007)

2. Eppinger, S., Browning, T.: Design Structure Matrix Methods and Applications. The MIT Press (2012)

3. Kelly, G.A.: The psychology of Personal Constructs, vols. 1 and 2. Norton, New York (1995)

4. Ohtomi, K.: Design of Worth for Consumer Product Development. In: Special issue Of Japanese Society for the Science of Design 2009, pp. 31-38 (2009)

5. Ohtomi, K., Hosaka, R.: Design for Product Sound Quality. In: INTER-NISE and NOISECON Congress and Conference Proceedings. Institute of Noise Control Engineering 2008, pp. 2257-2264 (2008)

6. Seki, K., Nishimura, H., Ishii, K., Balmelli, L.: Thermal/Acoustic trade-off design for Consumer Electronics in a distributed design environment. The International Council on Systems Engineering (INCOSE 2009) (2009), 0723.pdf

7. Yamagishi, K., Ohtomi, K., Seki, K., Nishimura, H.: The Sound design process using the use-case driven requirement and functional modelling. The Japan Society of Mechanical Engineers Annual Meeting 2013 (2013) (in Japanese)

8. Yanagisawa, H., Kataoka, A., Murakami, T., Ohtomi, K., Hosaka, R.: Extraction of latent emotional factors by analyzing human sensitivity towards unexplored design: Application to product sound design. In: Proceedings of the 17th International Conference on Engineering Design (ICED 2009), vol. 7 (2009)

9. Zhu, S., Nishimura, H., Balmelli, L.: System Integration of Motorcycle Driving Stability Control Using SysML. In: Proceedings of 3rd Asia-Pacific Conference on Systems Engineering, pp. 1-10 (2009) 